\title{
Potent anti-inflammatory agent escin does not affect the healing of tibia fracture and abdominal wound in an animal model
}

\author{
LEIMING ZHANG ${ }^{*}$, HONGSHENG WANG* ${ }^{*}$ TIAN WANG, NA JIANG, \\ PENGFEI YU, FEIYAN LIU, YATING CHONG and FENGHUA FU
}

\author{
Department of Pharmacology, School of Pharmacy, Yantai University, Yantai, Shandong 264005, P.R. China
}

Received December 14, 2011; Accepted January 19, 2012

DOI: $10.3892 /$ etm.2012.467

\begin{abstract}
Escin, a potent anti-inflammatory and anti-edematous agent, has been widely used clinically in preventing inflammatory edema after trauma, such as fracture and surgery. The aim of this study was to investigate whether escin has an inhibitory effect on fracture healing, and whether escin has an inhibitory effect on wound healing after surgery. Male New Zealand white rabbits underwent tibial mid-diaphyseal osteotomy, and were administered escin once per day for 10 days. At weeks 2, 4 and 6, bone fracture healing and bone mineral density were measured. The histologic examination of callus, osteocalcin, alkaline phosphatase, calcium and phosphate in the serum were also assayed. In another experiment, the rats underwent midline laparotomy, and received escin once prior to or after the operation. Six days later, the abdominal incision wounds were excised for measuring hydroxyproline levels. The results showed that there were no significant differences in fracture healing between the model and rabbits administered escin, and escin did not affect the hydroxyproline levels in the abdominal incision wounds of the rats. These findings suggest that escin has no inhibitory effect on fracture and wound healing in animal models.
\end{abstract}

\section{Introduction}

Escin is the main active constituent of Aesculus hippocastanum seed extracts, which is a triterpene saponin mixture consisting of A, B, C and D escin. Accumulating experimental evidence in previous studies suggests that escin exerts potent anti-inflammatory and anti-edematous effects. Escin inhibits acetic acid-induced increase in capillary permeability and adhesion formation in animal models (1). Escin also attenuates hippocampal injury after global cerebral ischemia in mice

Correspondence to: Dr Fenghua Fu, Department of Pharmacology, School of Pharmacy, Yantai University, Yantai, Shandong 264005, P.R. China

E-mail: fufenghua@sohu.com

"Contributed equally

Key words: escin, fracture healing, wound healing, rabbit, rat via regulating certain inflammatory genes (2). A recent study showed that escin has a potent protective effect on LPS-induced acute lung injury by inhibiting the inflammatory response (3).

According to Matsuda et al (4), the anti-inflammatory effects of escin are mainly dependent on its anti-histaminic and anti-serotoninergic activities. Another study (5) reported that escin dose-dependently prevented the hypoxia-induced activation of human endothelial cells, as evidenced by the inhibition of hypoxia-increased phospholipase A2, an enzyme responsible for the release of precursors of inflammatory mediators. In addition, escin significantly inhibited nuclear factor $(\mathrm{NF})-\kappa \mathrm{B}$ activation and down-regulated the expression of tumor necrosis factor (TNF)- $\alpha$, alleviating brain edema in traumatic brain injured rats (6). The experiments listed above characterized that escin has potent anti-inflammatory effects and its anti-inflammatory mechanisms are similar to glucocorticoids (GCs). Furthermore, we recently found that escin exerts synergistic anti-inflammatory effects with glucocorticoids (7), and its mechanism involves the up-regulation of the glucocorticoid receptor (GR) (3).

In China, escin has been widely used clinically in preventing inflammatory edema after trauma, such as fracture and surgery (8-10). However, it is unclear whether escin affects fracture healing, and whether escin has an inhibitory effect on wound healing. In the present study, we investigated the direct effects of escin on tibia fracture healing and abdominal wound healing in rabbit and rat models, respectively.

\section{Materials and methods}

Chemicals and instruments. Sodium salt of escin (i.e., sodium escinate, consisting of $\mathrm{A}, \mathrm{B}, \mathrm{C}$ and $\mathrm{D}$ and containing at least 65\% A and B) (batch no. 080902) was supplied by Shandong Luye Pharmaceutical Co., Ltd. (Yantai, China). Osteocalcin was supplied by R\&D Systems (batch no. CK-E90207R). Alkaline phosphatase (ALP), hydroxyproline, calcium and phosphate test kits were purchased from the Institute of Nanjing Jiancheng Bioengineering (Nanjing, China). All other chemicals and reagents used in this study were of analytical grade.

Animals. New Zealand white rabbits weighing $3 \pm 0.5 \mathrm{~kg}$ were provided by the Limited Liability Company of Luzhou (Anqiu, Shandong, China), and the certicate number was 
G080710. Female Sprague-Dawley rats weighing 250-300 g were provided by the Experimental Animal Center of Luye Pharmaceutical Company. All experimental procedures carried out in this study were performed in accordance with the Guidelines for the Care and Use of Laboratory Animals of Yantai University, and were approved by the ethics committee. The animals were housed in a temperature-controlled room $\left(22 \pm 2^{\circ} \mathrm{C}\right)$ on a 12 -h light/dark cycle with free access to food and water.

Surgical procedure and experimental design. Rabbits were anesthetized by subcutaneous administration of urethane $(1.6 \mathrm{~g} / \mathrm{kg})$, and placed in a lateral position on the operation table. The right tibia was approached through a $4-\mathrm{cm}$ long skin incision. The fascia was cut, the muscles separated and the posterior medial surface of the tibia was opened. The periosteum was incised longitudinally and retracted. With a stainless steel plate $(26 \times 6 \times 1 \mathrm{~mm})$ adhering to the side surface of the tibia, 4 holes were drilled with a $1.2-\mathrm{mm}$ drill. The periosteum was protected and an osteotomy was performed at the mid-diaphysis between the second and third holes. The steel plate and 4 stainless steel self-taping screws $(1.1 \times 10 \mathrm{~mm})$ were used as the inner fixator, while 4 wooden splints were used as the external fixator. The wound was irrigated with sterile normal saline and closed in layers with interrupted sutures. Postoperative radiographs were used to confirm the quality of the osteotomy. After the operation, the rabbits were randomly divided into four groups with 6 animals in each group. From the day of operation, animals in the model group were injected with saline via the marginal ear vein once per day for 10 days, while the animals in the other groups were injected with escin $(0.225,0.45$ or $0.9 \mathrm{mg} / \mathrm{kg})$ via the marginal ear vein, and the escin dosage was determmined according to the therapeutic dose for inflammatory edema treatment in the clinic.

Fifty rats were randomly divided into five groups: control group, model group, escin $1.8 \mathrm{mg} / \mathrm{kg}$ group (escin administered $6 \mathrm{~h}$ before abdominal incision, $-6 \mathrm{~h}$ ), escin $1.8 \mathrm{mg} / \mathrm{kg}$ group (escin administered just after abdominal incision, $0 \mathrm{~h}$ ) and escin $1.8 \mathrm{mg} / \mathrm{kg}$ group (escin administrated $24 \mathrm{~h}$ after abdominal incision, $+24 \mathrm{~h}$ ). The model and escin administration rats were anesthetized intraperitoneally with chloral hydrate $(400 \mathrm{mg} / \mathrm{kg})$, and placed on the operation table. After the abdominal skin was shaved with a povidone-iodine scrub, a $4-\mathrm{cm}$ long midline laparotomy was performed. The abdominal fascia and skin were subsequently closed by silk sutures. No surgery was performed in the control group rats. Six days after the operation, animals in each group were sacrificed. The abdominal incision wounds $(2 \times 1 \mathrm{~cm}$ portion of the abdominal wall sample) were excised and stored at $-80^{\circ} \mathrm{C}$ for measuring the hydroxyproline levels.

Radiographic evaluation. Both anterior-posterior and lateral $\mathrm{X}$-rays of the right tibia were performed at 2, 4 and 6 weeks to study callus formation and fracture healing using the VR X-ray shoot apparatus (Fairfield, CT, USA).

Bone mineral density. With the rabbits under anesthetization induced by urethane $(0.8 \mathrm{~g} / \mathrm{kg})$, the right tibia was scanned by a dual-energy X-ray absorptiometry (DEXA) scanner (Lunar DPX, USA) in a standard position with the lateral surface of the bone facing the scanner plate. At 2, 4 and 6 weeks, the bone mineral density (BMD) value of a $15-\mathrm{mm}$ long and 30-mm wide 'ROI' was measured centrally between the second and the third screws, which included the proximal and distal old bone and the callus.

Histological analysis. One rabbit selected from each group was sacrificed at 4 and 8 weeks after operation, respectively. Retrievals consisting of the $3-\mathrm{mm}$ segmental defect and $7 \mathrm{~mm}$ of cortical bone proximal and distal with the corresponding tibia, adjacent to the defect, were fixed in $4 \%$ paraformaldehyde at $4^{\circ} \mathrm{C}$ for $24 \mathrm{~h}$. After fixation, bones were rinsed in phosphate-buffered saline (PBS). The tissues were then decalcified in $5 \% \mathrm{HCl}$ buffered formaldehyde at $4^{\circ} \mathrm{C}$ for 2 weeks, dehydrated in ascending concentrations of ethanol and embedded in paraffin. During embedding, the positioning of the tibia was standardized in an attempt to ensure that the same region was evaluated in all specimens. Sections $(\sim 4-\mu \mathrm{m})$ were obtained at the middle of the specimens on a Polycut microtome (Leica, CM1950, Germany) and stained with H\&E. Pathological observation of the tissues was performed under light microscopy.

Serum levels of osteocalcin, ALP, calcium and phosphate. On day 3, and weeks 1, 2 and 4 post-surgery, blood was obtained via the ear vein and centrifuged at $10,000 \mathrm{x} g$ for $10 \mathrm{~min}$. The plasma was then analyzed for osteocalcin, ALP, calcium and phosphate concentration using the corresponding kit.

Measuring hydroxyproline. The abdominal wall samples were homogenized with ice-cold saline for a $10 \%(w / v)$ homogenate. The hydroxyproline contents were determined according to the method of Bergman and Loxley (11) as $\mathrm{mg}$ per $100 \mathrm{mg}$ of tissue.

Statistical analysis. One-way ANOVA was used to analyze the significant differences within the different groups; the comparison between two groups was determined by the Student's unpaired t-test, using SPSS 11.5 statistical software. $\mathrm{p}<0.05$ was accepted as indicative of a statistical significant difference among the groups. All data in this study were expressed as the mean values \pm SD.

\section{Results}

Effects of escin on the healing of fracture. Two weeks postoperation, the fracture gap remained visible and the callus was difficult to be recognized in all groups. By contrast, at 4 weeks the callus size was markedly larger and the fracture gap became less obvious, while there was no significant difference among all groups. After 6 weeks, calluses in all groups were apparent (Fig. 1). However, no significant difference was found between the escin-treated groups and the model group.

Effects of escin on the bone mineral density after fracture. At weeks 2, 4 and 6, no significant differences were found between the model group and the escin-treated groups (Fig. 2).

Effects of escin on the histology of bone fracture. Histological examination at week 4 after the operation demonstrated a 

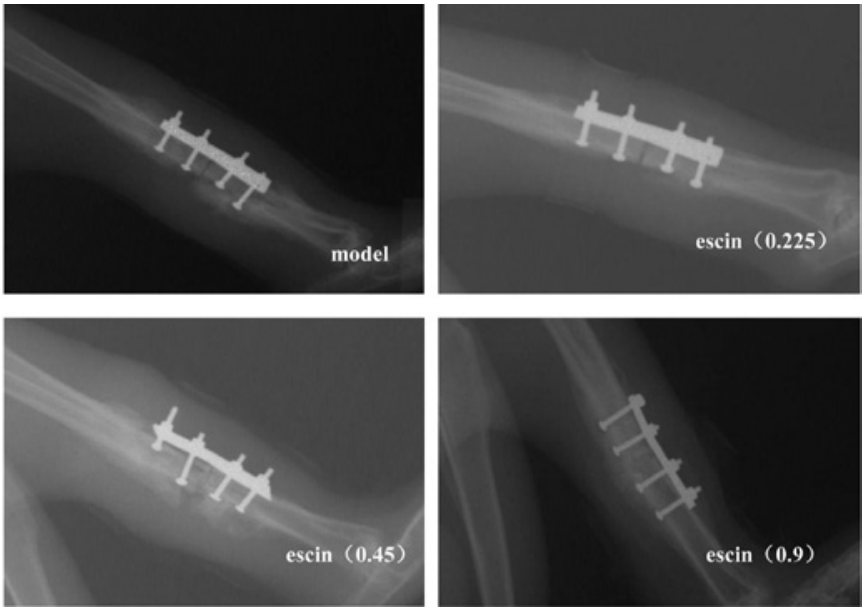

Figure 1. Radiographs of the right tibia of rabbits taken at 6 weeks after osteotomy. Calluses in all groups were apparent. No significant differences in calluses were observed between the escin-treated groups and the model group.

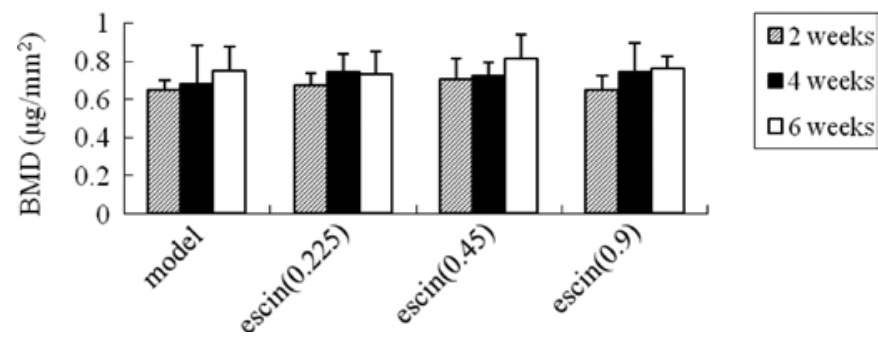

Figure 2. Effect of escin on bone mineral density (BMD) values of the fracture site at 2, 4 and 6 weeks after osteotomy. A DEXA (Dual-energy X-ray absorptiometry) scanner was used to measure the BMD value of a $15-\mathrm{mm}$ long and 30-mm wide 'ROI' centrally between the second and third screws, which included the proximal and distal old bone and the callus. No significant difference was found among groups.

large number of fibroblasts and chondrocytes both in mature and immature stages, while no significant difference was observed among all groups (Fig. 3A). Histology at week 8 post-operation showed also no significant difference among groups with respect to cartilage cells, mature bone cells, bone lamella and haversian system (Fig. 3B).

Effects of escin on the serum osteocalcin and phosphatase alkaline after fracture. One week after the operation, the serum osteocalcin levels in the escin-treated group $(0.45 \mathrm{mg} / \mathrm{kg})$ was higher than that in the model group $(\mathrm{p}<0.05)$, while no significant difference was found between the treated groups and the model group at other time intervals (i.e., day 3 and weeks 2 and 4 post-operation) (Fig. 4A).

At different time intervals (i.e., day 3 and weeks 1, 2 and 4 post-operation), the phosphatase alkaline levels of the treated groups were comparable to that of the model group, and the differences were not statistically significant (Fig. 4B).

Effects of escin on serum calcium and phosphate levels after fracture. One week after the operation, the serum calcium levels in the escin-treated groups $(0.45$ and $0.9 \mathrm{mg} / \mathrm{kg})$ were higher compared to the model group $(\mathrm{p}<0.05)$. At other time intervals (i.e., day 3 and weeks 2 and 4 after the operation)
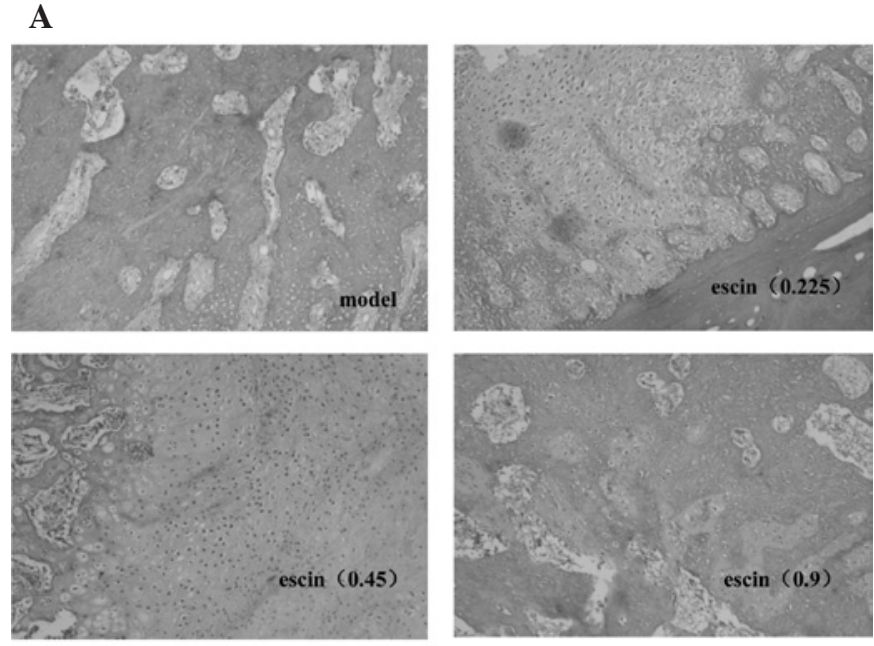

B
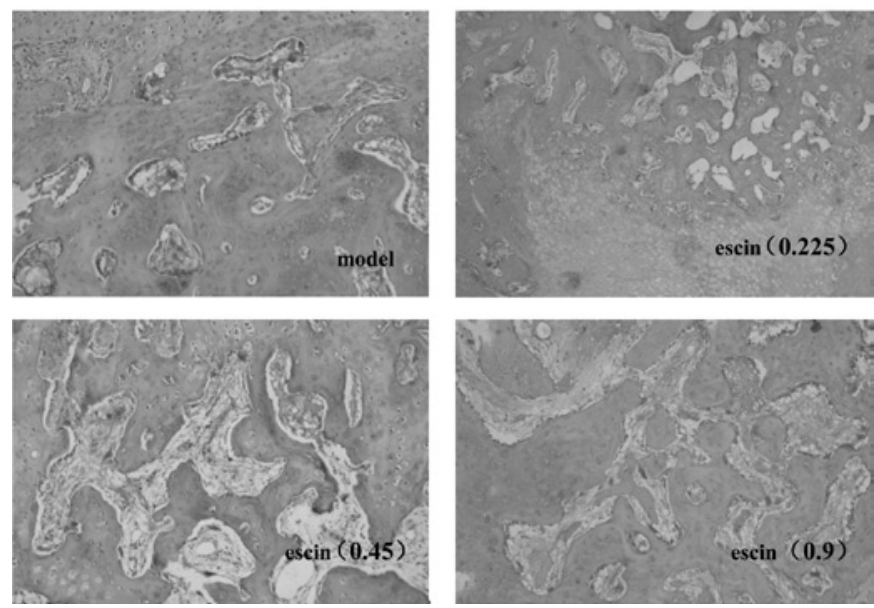

Figure 3. Histological sections through the tibia osteotomy callus at 4 and 8 weeks after the operation. (A) Four weeks after the operation, a large number of fibroblasts and chondrocytes in mature and immature stages are noted. (B) Eight weeks post-operation, bone cells reached maturity, while the haversian system and a small number of cartilage cells are noted. There was no significant difference among groups.

however, no significant difference was observed between the treated groups and the model group (Fig. 4C). On the other hand, at week 2 after the operation, the serum phosphate levels of the escin-treated group $(0.9 \mathrm{mg} / \mathrm{kg})$ were lower than those of the model group $(\mathrm{p}<0.05)$, while no significant difference was noted between the treated groups and the model group at other time intervals (Fig. 4D).

Effects of escin on the hydroxyproline levels after the operation. The hydroxyproline content of the abdominal wall samples in the model group was higher compared to that in the control group $(\mathrm{p}<0.01)$. However, there were no significant differences between the escin-administered and the model groups (Fig. 5).

\section{Discussion}

Glucocorticoids exhibit excellent anti-inflammatory and anti-edematous effects, and have been widely used clinically to prevent inflammatory edema after trauma. However, glucocorticoids exhibit multiple effects to inhibit the immune 

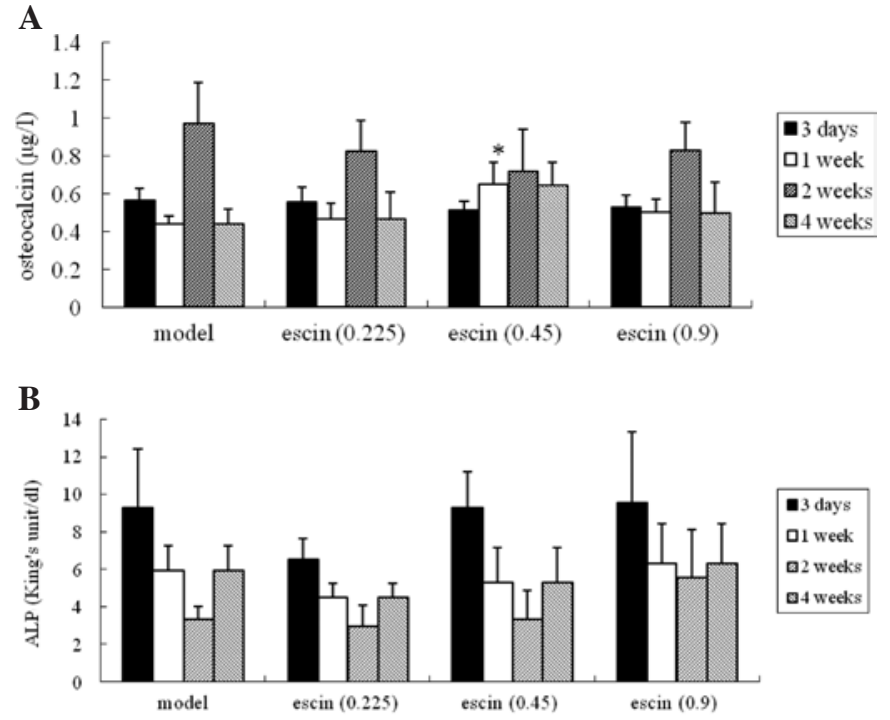

C

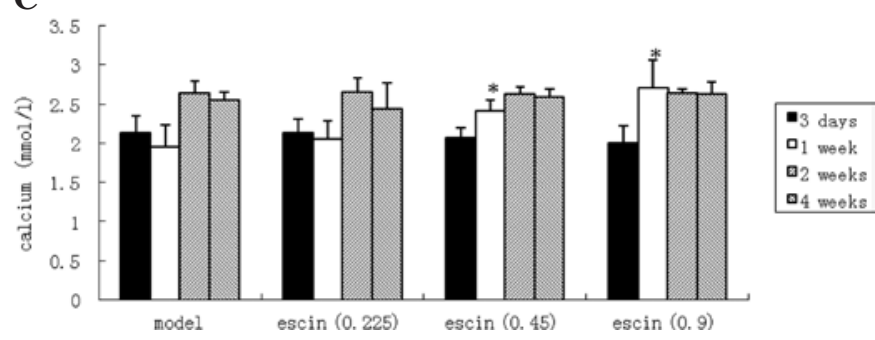

D

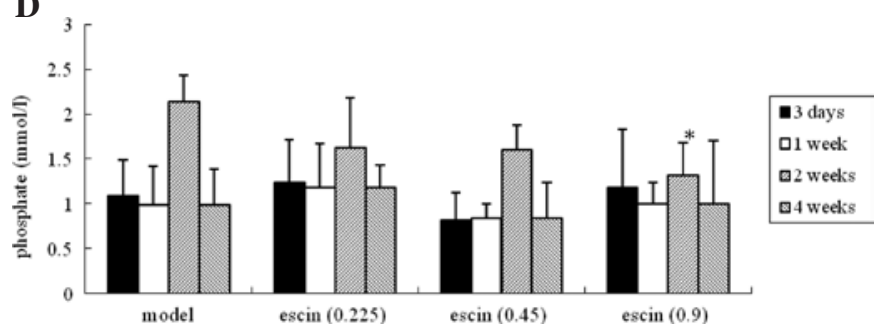

Figure 4. Effect of escin on the serum contents of osteocalcin, ALP, calcium and phosphate. (A) One week after the operation, the serum levels of osteocalcin in the escin-treated group $(0.45 \mathrm{mg} / \mathrm{kg})$ were significantly higher than those of the other groups $(\mathrm{p}<0.05)$. (B) No significant differences were found between the treated groups and the model group with respect to the serum levels of ALP, (C) while the calcium levels in the escin groups $(0.45$ and $0.9 \mathrm{mg} / \mathrm{kg}$ ) were higher than that in the model group ( $<<0.05$ ). (D) Two weeks after the operation, the concentration of serum phosphate in the escin group $\left(0.9 \mathrm{mg} / \mathrm{kg}\right.$ ) was lower than that in the model group $(\mathrm{p}<0.05)$. ${ }^{\mathrm{p}}<0.05$ compared to the model group.

system, and are also associated with a risk for osteoporosis and an increased susceptibility to reduced wound healing (12). Accumulating experimental evidence in previous studies suggests that escin exerts potent anti-inflammatory and antiedematous effects, such as inflammatory edema after fracture. However, it is unclear whether escin affects fracture healing and whether escin has an inhibitory effect on wound healing. In the present study, we investigated the direct effects of escin on tibia fracture healing and abdominal wound healing in rabbit and rat models, respectively, and the dosage of escin was determined according to the therapeutic dose for inflammatory edema treatment in the clinic.

In order to study the fracture healing process, many methods including the radiological and histological findings,

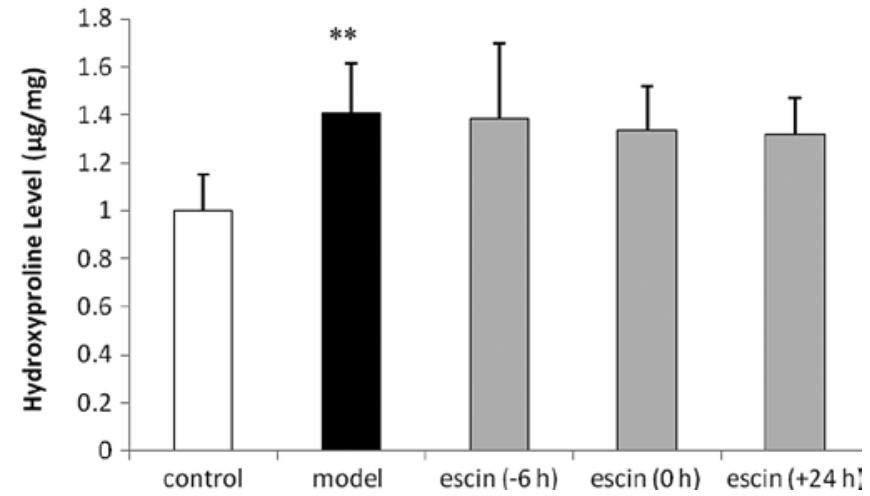

Figure 5. Effect of escin on hydroxyproline tissue content of the abdominal wound in rats. The hydroxyproline tissue content of the abdominal wall samples in the model group was higher than that of the control group $(p<0.01)$. However, there were no significant differences between the escinadministered groups and the model group. ${ }^{* *} \mathrm{p}<0.01$ compared to the control.

DEX absorptiometry (13-16) and serum determination of osteocalcin, phosphatase alkaline, calcium and phosphate $(17,18)$ have been used.

Radiographic follow-up provides a visual evaluation and monitoring of regenerative tissue formation. The callus size and fracture gap reflect the extent of bone healing $(13,15,19)$. In our study, no significant difference was observed between the escin-treated groups and the model group. This indicates that escin does not affect the progress of bone healing.

BMD values reflect the mineralization of bone healing, which is important for the strength and function of the bone (13-15). In this study, no significant difference was observed between the escin-treated and the model rabbits. The results indicate that the administration of escin does not affect the mineralization during the fracture healing process.

The physiological process of fracture healing can be summarized as follows $(20,21)$. A few hours after fracture, the extravascular blood cells form a blood clot, known as a hematoma. Then, fibroblasts replicate and form granulation tissue. Days after fracture, the periosteal cells replicate and develop into chondroblasts and osteoblasts, which form hyaline cartilage and woven bone. The fibroblasts within the granulation tissue develop into chondroblasts which also form hyaline cartilage. Subsequently, the hyaline cartilage and woven bone are replaced with lamellar bone and the haversian system takes shape. Meanwhile, the osteoblasts form new lamellar bone upon the recently exposed surface of the mineralized matrix. This new lamellar bone is in the form of trabecular bone. Eventually, the trabecular bone is replaced by compact bone. Therefore, the amount of fibroblasts, chondroblasts and osteoblasts, the area of hyaline cartilage, woven bone, lamellar bone and the thickness of bone trabeculae show the extent of fracture healing $(14,16,19)$. In the present study, 4 weeks after the operation, a large number of fibroblasts and chondrocytes in mature and immature stages were found. Eight weeks post-surgery, bone cells reached maturity, while the haversian system and a small number of cartilage cells were observed. However, there was no significant difference among groups, which again confirms that escin does not affect the fracture healing process. 
In humans, ALP is produced mostly in the liver and bone. Elevated ALP indicates that there may be active bone formation occurring as ALP is a by-product of osteoblast activity (22). In our study, no significant difference was found between the treated groups and the model group, with respect to the serum levels of ALP, which demonstrated that escin does not affect osteoblast activity. Osteocalcin is synthesized by osteoblasts, and a small fraction of the newly synthesized protein is released into the circulation where it can be measured (23). As osteocalcin is bone-specific, it has been widely used as a marker of bone formation (24). Calcium and phosphate are important components of bone matrix, and their serum contents directly reflect the metabolism of bone (18). In our study, 1 week after the operation, the serum osteocalcin levels in the escin-treated group $(0.45 \mathrm{mg} / \mathrm{kg})$ were significantly higher than that in the model group $(\mathrm{p}<0.05)$, while the calcium levels in the escintreated groups $(0.45$ and $0.9 \mathrm{mg} / \mathrm{kg})$ were higher than that in the model group $(\mathrm{p}<0.05)$. The data showed that the treatment with escin increased the calcium and osteocalcin contents in the serum, while no significant difference was found between the escin-treated groups and the model group at other time intervals; the mechanism remains unclear and further study is required.

Wound healing is the process of repair that follows injury to the skin and other soft tissues. A number of factors regulate wound repair. Hydroxyproline is an amino acid and a subproduct of collagen synthesis. The tissue hydroxyproline assay presents a parallel increase with the tissue collagen level; therefore, hydroxyproline measurement is an important test for wound healing $(11,25)$. In the present study, the hydroxyproline levels showed a significant increase in the model rat group. However, there were no significant differences between the escin-administered rat groups and the model rat group. The results revealed that escin did not affect the wound healing of the abdominal wall in rats.

In conclusion, according to the present study escin does not affect the process of fracture healing and wound healing, which provides rational evidence for using escin after fracture in the clinic.

\section{Acknowledgements}

This study was supported by Taishan Scholar Project, the 11th Five Years Key Programs for Science and Technology Development of China (grant no. 2008ZX09202-008), and the National Natural Science Foundation of China (grant no. 30772760). The authors would like to thank Professor Tongshen Liu and all the staff of the Health Examination Center of the Yantai Mountain Hospital for their technical assistance.

\section{References}

1. Fu F, Hou Y, Jiang W, Wang R and Liu K: Escin: inhibiting inflammation and promoting gastrointestinal transit to attenuate formation of postoperative adhesions. World J Surg 29: $1614-1620,2005$

2. Zhang L, Fu F, Zhang X, Zhu M, Wang T and Fan H: Escin attenuates cognitive deficits and hippocampal injury after transient global cerebral ischemia in mice via regulating certain inflammatory genes. Neurochem Int 57: 119-127, 2010.
3. Xin W, Zhang L, Fan H, Jiang N, Wang T and Fu F: Escin attenuates acute lung injury induced by endotoxin in mice. Eur J Pharmaceut Sci 42: 73-80, 2011.

4. Matsuda H, Li Y, Murakami T, Ninomiya K, Yamahara J and Yoshikawa M: Effects of escins Ia, Ib, IIa, and IIb from horse chestnut, the seeds of Aesculus hippocastanum L., on acute inflammation in animals. Biol Pharmaceut Bull 20: 1092-1095, 1997.

5. Arnould T, Janssens D, Michiels C and Remacle J: Effect of aescine on hypoxia-induced activation of human endothelial cells. Eur J Pharmacol 315: 227-233, 1996.

6. Xiao GM and Wei J: Effects of $\beta$-aescin on the expression of nuclear factor-kappa B and tumor necrosis factor-alpha after traumatic brain injury in rats. J Zhejiang Univ Sci B 6: 28-32, 2005.

7. Xin W, Zhang L, Sun F, et al: Escin exerts synergistic antiinflammatory effects with low doses of glucocorticoids in vivo and in vitro. Phytomedicine 18: 272-277, 2011.

8. Ma CF and Li L: Clinical observation of the curative effects of a combination of sodium aescinate and glycerol fructose on joint swelling after calcaneal fracture. China Pharmacist 11: 445-446, 2008.

9. Tang L: Clinical observation of the curative effects of sodium aescinate on swelling after ankle fracture. Mod Med J 36: 105-106, 2008

10. Yang LD and Liu F: Clinical observation of the curative effects of sodium aescinate on limb swelling resulting from fracture of tibia and fibula. Acta Academiae Medicinae Nantong 29: 34-35, 2009.

11. Bergman I and Loxley R: New spectrophotometric method for the determination of proline in tissue hydrolyzates. Anal Chem 42: 702-706, 1970.

12. Christian LM, Graham JE, Padgett DA, Glaser R and KiecoltGlaser JK: Stress and wound healing. Neuroimmunomodulation 13: 337-346, 2006.

13. Wang CJ, Yang KD, Wang FS, Hsu CC and Chen HH: Shock wave treatment shows dose-dependent enhancement of bone mass and bone strength after fracture of the femur. Bone 34 : 225-230, 2004.

14. Morgan EF, Mason ZD, Bishop G, et al: Combined effects of recombinant human BMP-7 (rhBMP-7) and parathyroid hormone (1-34) in metaphyseal bone healing. Bone 43: 1031-1038, 2008.

15. Aleksyniene R, Thomsen JS, Eckardt H, Bundgaard KG, Lind M and Hvid I: Parathyroid hormone PTH (1-34) increases the volume, mineral content, and mechanical properties of regenerated mineralizing tissue after distraction osteogenesis in rabbits. Acta Orthopaedica 80: 716-723, 2009.

16. Saghieh S, Khoury NJ, Tawil A, et al: The impact of zoledronic acid on regenerate and native bone after consolidation and removal of the external fixator: an animal model study. Bone 46: 363-368, 2010

17. Taniguchi T, Matsumoto T and Shindo H: Changes of serum levels of osteocalcin, alkaline phosphatase, IGF-I and IGF-binding protein-3 during fracture healing. Injury 34: 477-479, 2003.

18. Andreen $\mathrm{O}$ and Larsson SE: Effects of 1,25-dihydroxycholecalciferol on fracture healing. Calcium, phosphate, and zinc in callus and serum. Arch Orthop Trauma Surg 103: 257-262, 1984.

19. O'Connor JP, Capo JT, Tan V, Cottrell JA, Manigrasso MB, Bontempo N and Parsons JR: A comparison of the effects of ibuprofen and rofecoxib on rabbit fibula osteotomy healing. Acta Orthopaedica 80: 597-605, 2009

20. Brighton CT and Hunt RM: Early histological and ultrastructural changes in medullary fracture callus. J Bone Joint Surg 73: 832-847, 1991.

21. Brighton CT and Hunt RM: Early histologic and ultrastructural changes in microvessels of periosteal callus. J Orthop Trauma 11: 244-253, 1997.

22. Coleman JE: Structure and mechanism of alkaline phosphatase. Ann Rev Biophys Biomol Struct 21: 441-483, 1992.

23. Akesson K, Ljunghall S, Gärdsell P, Sernbo I and Obrant KJ: Serum osteocalcin and fracture susceptibility in elderly women. Calcif Tissue Int 53: 86-90, 1993.

24. Herrmann M, Klitscher D, Georg T, Frank J, Marzi I and Herrmann W: Different kinetics of bone markers in normal and delayed fracture healing of long bones. Clin Chem 48: 2263-2266, 2002.

25. Brown GL, Curtsinger LJ, White M, et al: Acceleration of tensile strength of incisions treated with EGF and TGF-beta. Ann Surg 208: 788-794, 1988. 